\title{
Assessment on the compressive strength behavior of hybrid filled epoxy nanocomposite
}

\begin{abstract}
Compressive strength of the epoxy matrix loaded by combination of hybrid filler is expected to benefit by the hollow nature of microsphere and highly porous silica aerogel and, consequently it also depends on the suitable ratio of microsphere:silica aerogel (Mic:SilAe). In this study, the morphology of the fillers was investigated by Scanning Electron Microscope (SEM) for microstructure analysis. The nanocomposite was then prepared by shear mixing technique. Compressive yield and fracture strength behavior were assessed as a function of Mic:SilAe ratios and its dispersion in epoxy matrix. The compressive yield and fracture strength increased monotonously with inclusion of considerable ratio of Mic:SilAe. The optimum loading of Mic:SilAe in epoxy nanocomposite was attained at filler ratio of 1:1 for compressive yield strength and filler ratio of 5:1 for compressive fracture strength, where the improvement were $28 \%$ and $90 \%$, respectively.
\end{abstract}

Keyword: Compressive; Epoxy; Nanocomposite; Silica aerogel; Microsphere 